\title{
Outcomes and Issues of 'Drip and Go' as Original Article an Inter-Hospital Cooperation System in Mechanical Thrombectomy for Acute Ischemic Stroke
}

Masahiro Nishihori, Takashi Izumi, Tetsuya Tsukada, Kinya Yokoyama, Kenji Uda, Yoshio Araki, and Toshihiko Wakabayashi

Objective: Mechanical thrombectomy in acute ischemic stroke (AIS) has become popular in recent years. Our affiliated institutes without neuro-endovascular specialists call our department to come to assist and perform thrombectomy (Drip and Go). In this study, the effectiveness of this inter-hospital cooperative system was evaluated.

Methods: Between January 2016 and December 2018, "Drip and Go" was performed in a total of 29 patients (20 males, average age of 75 years) from four hospitals located within a 1-hour drive, that frequently called for AIS assistance. The background and outcomes of such cases were then retrospectively collected and evaluated.

Results: The median National Institutes of Health Stroke Scale (NIHSS) and diffusion-weighed image-Alberta Stroke Programme Early CT Score (DWI-ASPECTS) were 19 and 7, respectively. Gro in puncture was performed in 27 patients $(93 \%)$ within $6 \mathrm{~h}$ of onset. Good reperfusion (thrombolysis in cerebral infarction [TICl] 2b/3) was obtained in 24 patients $(82 \%)$ with only one patient exhibiting hemorrhagic complication. A total of 12 patients $(41 \%)$ had a modified Rankin Scale (mRS) score of 0-3 after 90 days or at the time of discharge. Univariate analysis identified a DWI-ASPECTS of 7 or higher as the only significant factor associated with a good neurological prognosis $(P<0.05)$. Neurological prognosis was the most favorable at the furthest hospital where patients had a good DWI-ASPECTS.

Conclusion: By employing a 1-hour arrival time window and proper patient selection, the "Drip and Go" inter-hospital cooperative system can be an alternative approach for covering areas where no neuro-endovascular specialists are available for AIS.

Keywords $>$ acute ischemic stroke, mechanical thrombectomy, inter-hospital cooperation system, drip and go

\section{Introduction}

Mechanical thrombectomy for acute ischemic stroke (AIS) has become routine in Japan following improvements in recanalization rates thereafter. ${ }^{1,2}$ Previous studies have indicated that treatment outcomes depend on the interval between onset and recanalization. ${ }^{3,4)}$ The establishment of

Department of Neurosurgery, Nagoya University Graduate School of Medicine, Nagoya, Aichi, Japan

Received: May 23, 2020; Accepted: December 11, 2020 Corresponding author: Masahiro Nishihori. Department of Neurosurgery, Nagoya University Graduate School of Medicine, 65, Tsurumaicho, Showa-ku, Nagoya, Aichi 466-8550, Japan Email: nishihori@med.nagoya-u.ac.jp

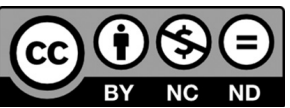

This work is licensed under a Creative Commons Attribution-NonCommercialNoDerivatives International License.

(C)2021 The Japanese Society for Neuroendovascular Therapy a medical cooperative system or in-hospital system for AIS management helps to shorten the time. ${ }^{5,6)}$ Several studies have compared "Mothership," herein patients who may be indicated for mechanical thrombectomy are directly transported to a comprehensive stroke center (CSC), with "Drip and Ship," wherein those who should be indicated for mechanical thrombectomy are transported after initial treatment. ${ }^{7-9)}$ A recent meta-analysis reported that functional independence was better in the Mothership group, whereas there were no significant differences in recanalization rate, incidence of hemorrhage, or mortality rate. ${ }^{10)}$ Recently, CSCs and thrombectomy-capable stroke centers (TSCs) have gradually emerged across Japan, although they have yet to be established in each secondary medical area. Furthermore, issues regarding manpower, hospital beds, and patient transportation remain. Considering that specialists in neuroendovascular treatment are not stationed in all core hospitals within a secondary medical 
area, patients are transported to peripheral core hospitals employing such specialists using the "Mothership" and "Drip and Ship" systems among our affiliated institutions. On the other hand, such specialists went to other hospitals for mechanical thrombectomy ("Drip and Go") from 2014 based on requests to our department from institutions in areas where transportation was difficult. Following the widespread recognition of the usefulness of mechanical thrombectomy, the number of treatment sessions has rapidly increased since 2018. Therefore, the current study sought to investigate treatment outcome and future issues of "Drip and Go," an inter-hospital cooperative system, although they may be markedly influenced by the stroke emergency system of each medical institution.

\section{Materials and Methods}

Between January 2016 and December 2018, 57 patients underwent mechanical thrombectomy at our department. Many of our affiliated institutions that lack specialists in neuroendovascular treatment have adopted the "Mothership" system for patients with AIS/large vessel occlusion (LVO). Overall, 29 patients from four institutions that adopted the "Drip and Go" system and continuously requested support were analyzed, excluding 28 in-university patients/those supported in the absence of full-time endovascular treatment specialists/those irregularly supported at institutions adopting the "Drip and Ship" system. Patient background data are summarized in Fig. 1. Devices, such as sheaths and guiding catheters, were installed at the affiliated hospitals, if possible, to save time. Physicians in charge of stroke at each affiliated hospital were responsible for initial treatment and tissue plasminogen activator (tPA) administration. Concerning the indication of mechanical thrombectomy, they consulted our department. A 24-hour system was adopted, with one or two neuroendovascular treatment specialists belonging to our department visiting the hospital for treatment by car or other means of transportation. Regarding procedures, a combined technique using a stent retriever (SR) and aspiration catheter was primarily selected. However, an SR or direct aspiration first pass technique (ADAPT) alone was used according to individual patients. As for the indication of treatment, we complied with an interval of $\leq 6$ hours from onset without establishing age or Alberta Stroke Programme Early CT Score (ASPECTS) limits to make flexible relationships with several medical institutions. This was an observational study, and its protocol was approved by the ethics review boards of our hospital and each affiliated institution.
Data on the patient background (age/sex/medical history/ modified Rankin Scale [mRS] score), details of stroke (site of occlusion/presence of tPA administration/National Institutes of Health Stroke Scale [NIHSS] score/computed tomography $[\mathrm{CT}] /$ magnetic resonance imaging [MRI]), treated site, treatment procedure/results, workflow time, and procedure-associated complications/mRS score after 90 days or on discharge as outcomes were retrospectively collected. Results were expressed as mean (standard deviation) or median (interquartile range). Values were then compared, establishing a cutoff NIHSS score as 26 , at which tPA should be carefully administered based on the guidelines for the adequate use of tPA in Japan. Concerning workflow time, the cutoff value of the puncture-to-reperfusion time (P2R) was established as 60 minutes according to the guidelines recommended by the Society of Neurointerventional Surgery (SNIS). ${ }^{11)}$ The cutoff value of the onset-to-puncture time (O2P) was established as approximately 270/438 minutes, which is associated with the mRS score and functional prognosis according to previous studies. ${ }^{12,13)}$ The chi-square test was used for data comparison. All statistical analyses were performed using EZR (R graphical user interface; Saitama Medical Center, Jichi Medical University, Saitama, Japan), with a $P$ value of 0.05 indicating statistical significance.

\section{Results}

The current study included four affiliated general hospitals that had $\geq 400$ beds (Hospitals A to D). Their positional relationships on a broad-area map around our university and the time required to arrive by car are presented in Fig. 2. The arrival time was determined using Google Maps, but it was similar to the actual time required. Hospital A is located in another prefecture and requires approximately 60 minutes of travel by car. On the other hand, Hospital D is located in Nagoya City, with favorable access, and it can be reached in approximately 20 minutes. Hospitals B and C are located in the suburbs of this prefecture, and approximately 40-50 minutes are required until arrival. Concerning the patient background, the mean age was 75 years, the subjects included 20 males (69\%), the median NIHSS score was 19 (16-24), and the diffusion-weighted image image-ASPECTS (DWI-ASPECTS) was 7 (5-9). Moreover, $90 \%$ of the subjects exhibited affected anterior circulation, while $60 \%$ of the patients received tPA. In 27 patients $(93 \%)$, puncture was performed within 6 hours after onset, with 24 patients (83\%) achieving favorable recanalization. The $\mathrm{mRS}$ score after 90 days (or on discharge) 


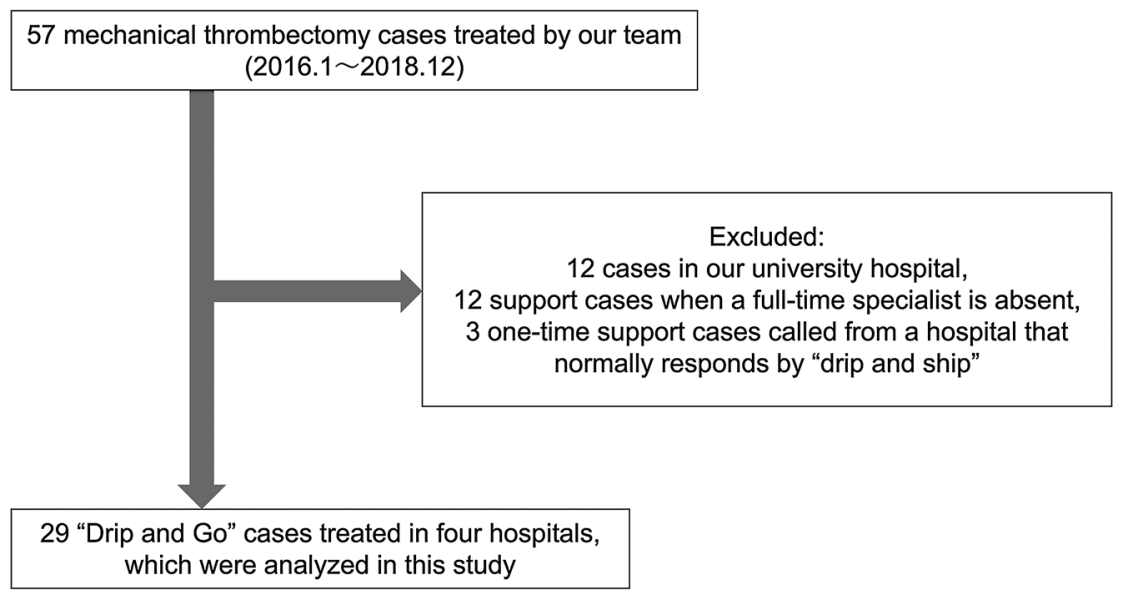

Fig. 1 Breakdown of AIS cases in which mechanical thrombectomy was performed within the period. AIS: acute ischemic stroke

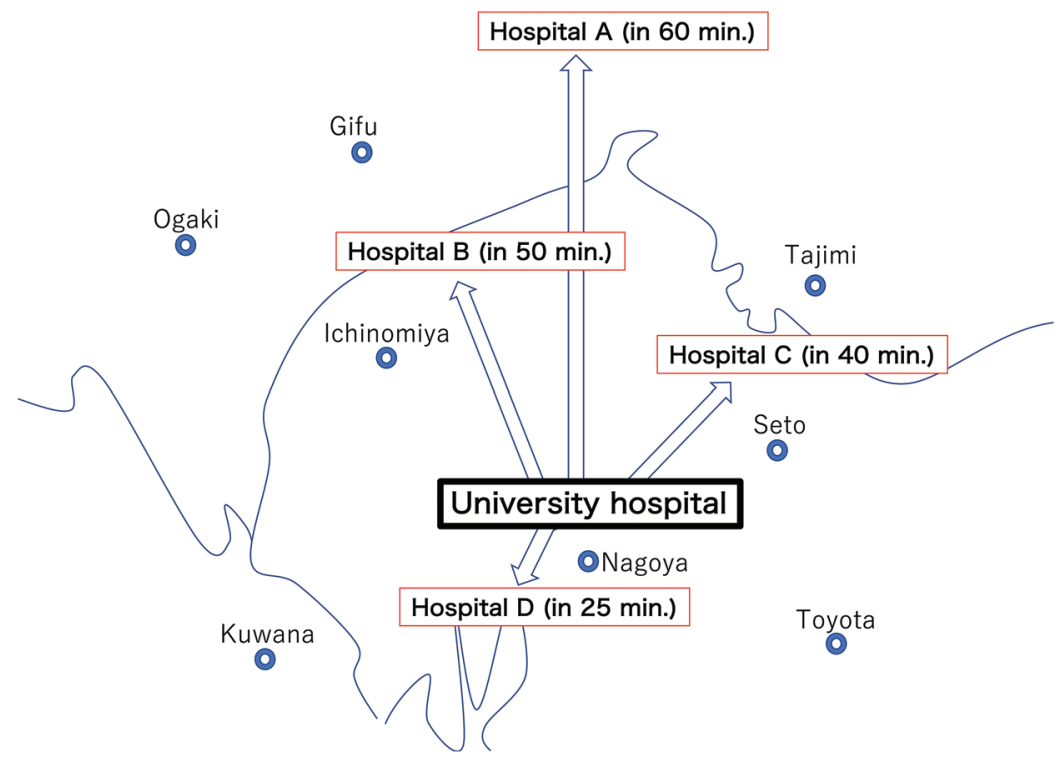

Fig. 2 Map showing the location of each hospital and the time required.

was $\leq 2$ in 6 patients $(21 \%)$, but it was $\leq 3$ in $12(41 \%)$. The pre-onset $\mathrm{mRS}$ score deteriorated by $\leq 2$ points in 11 patients $(38 \%)$.

\section{Differences in the patient background among the hospitals}

Table 1 summarized the clinical characteristics of the 29 patients who underwent mechanical thrombectomy. In Hospital D, there were a slightly higher proportion of elderly patients than the other hospitals. Treatment was performed even for low ASPECTS patients (median: 5). Overall, the median NIHSS score was 19, with Hospital B having a median score of 14 and Hospitals A and D having a median score of 22. Anterior circulation proximal areas (ICA/M1/ M2) were affected in $90 \%$ of occluded blood vessels.

\section{Interval until mechanical thrombectomy with the "Drip and Go" system and results of treatment}

The workflow time and results of mechanical thrombectomy are presented in Table 2. As our team was responsible for treatment, the devices used in individual patients differed, but a combined technique using aspiration catheter and SR was adopted in $80 \%$ of the subjects. The mean $\mathrm{O} 2 \mathrm{P}$ and $\mathrm{D} 2 \mathrm{P}$ in Hospitals $\mathrm{A}$ and $\mathrm{B}$, which were remote from our hospital, with no device installed, were approximately 290 and $210 \mathrm{~min}$, respectively, being prolonged. On 
the other hand, devices were installed in Hospital C, to which the distance is similar to that to Hospital B, with a 10-minutes difference; therefore, the $\mathrm{O} 2 \mathrm{P}$ and $\mathrm{D} 2 \mathrm{P}$ were approximately 60 minutes shorter than for Hospital B. The mean P2R was approximately 100 minutes for Hospital A, which is located in another prefecture, and is furthest from our hospital, considering that it had the longest interval until devices arrived. The mean P2R was similar among Hospitals B, C, and D. The median number of passes was 2. Thrombolysis in cerebral infarction (TICI) $2 \mathrm{~b}$ or higher recanalization was achieved in 24 patients (83\%), among whom 14 (48\%) achieved, first-pass recanalization was achieved. At each institution, recanalization was achieved in $\geq 70 \%$ of the patients. Hemorrhagic complication related to the procedure was observed in only one patient $(3 \%)$.

\section{Factors associated with the neurological prognosis}

This study analyzed the influence of background factors, such as age/NIHSS score/pre-onset mRS score, and workflow time on the postoperative mRS score. The indication for treatment was herein determined based on the interval from onset without strictly considering imaging findings. Therefore, this study conducted univariate analysis to determine patients who achieved favorable results (i.e., those with an mRS score of 0-3), with the results being presented in Table 3. The NIHSS score was approximately 26 , with no significant difference. Univariate analysis identified a DWI-ASPECTS of $\geq 7$ a factor significantly associated with good prognosis. Considering the limited number of patients, multivariate analysis could not be performed. Comparing the results among the four institutions showed that Hospital A had a high proportion of patients with favorable results (mRS 0-3: 67\%), whereas Hospital D, which is the closest to our hospital, had relatively poor results (mRS 0-3: 22\%).

\section{Discussion}

In 2016, the results of randomized controlled trials (RCTs), including the MRCLEAN, ${ }^{14)}$ and meta-analyses, such as the HERMES, ${ }^{12)}$ were published. Since then, the number of consultations on mechanical thrombectomy for LVO and treatment requests from affiliated institutions where specialists in neuroendovascular treatment are absent has increased. This study retrospectively analyzed patients who underwent mechanical thrombectomy at four institutions that previously requested us for treatment using the "Drip and Go" system between 2016 and 2018. Overall, our results demonstrated that the DWI-ASPECTS was better associated with a favorable neurological prognosis more than time-related factors when the inter-hospital transfer time was $\leq 1 \mathrm{~h}$, although the interval until recanalization increased for hospitals located farther away. Similarly, the transportation time may not be important even when adopting the "Mothership" system involving patient transportation to TSCs. In this system, treatment can be completed at the core hospital of an area and it is not necessary to transport stroke patients with symptoms to a distant medical institution, reducing patients' and their families' physical/ mental stress, which is advantageous. On the other hand, in the "Mothership" system in which patients are transported to TSCs or CSCs, the emergency-room (ER) and angiography-room staff are skilled at emergency mechanical thrombectomy, shortening the pre- and post-thrombectomy time and improving the nerve function prognosis through high-quality perioperative management. ${ }^{10,15-17)}$

\section{Treatment results in our series}

The basic procedure, utilized herein involved a combined technique with an aspiration catheter and SR in $80 \%$ of the subjects, with a median procedure time (P2R) of $68 \mathrm{~min}$. TICI $2 \mathrm{~b}$ or higher recanalization was achieved in 24 patients (83\%), among whom 14 (48\%) achieved first-pass recanalization. Recently, a trial involving the use of an SR suggested the usefulness of first-pass effect (FPE)-mediated recanalization. Such recanalization accounted for $51 \%-$ $68 \%$. In our series, a similar result was also obtained and there may have been no technical problem due to a low incidence of complications. A sub-analysis of the STRATIS registry revealed that the FPE was significantly more potent in the $40-\mathrm{mm}$ Solitaire group. ${ }^{18)}$ We also adopted a strategy to use a long SR. Furthermore, the nerve function prognosis in our series was poor considering that five patients had a preoperative $\mathrm{mRS}$ score $\geq 3$ and 10 patients with a low DWI-ASPECTS $(\leq 6)$ were included. However, when focusing on a group that meets the Grade A criteria recommended in the guidelines for the adequate use of devices for mechanical thrombectomy in Japan 1$)(n=17$; anterior circulation, DWI-ASPECTS: $\geq 7$, pre-onset $\mathrm{mRS}$ score: 0 or 1$)$, the $\mathrm{mRS}$ score was 0 to 2 in 6 patients (35\%), and it was 0 to 3 in 12 (71\%); the results were better than those of the HERMES. ${ }^{12)}$

\section{Influence of the inter-hospital transfer time}

As the number of "Drip and Go" patients has gradually increased, we retrospectively analyzed the treatment 
Table 1 Baseline clinical characteristics of "Drip and Go" patients at each hospital

\begin{tabular}{|c|c|c|c|c|c|}
\hline & $\begin{array}{l}\text { All cases } \\
(n=29)\end{array}$ & $\begin{array}{l}\text { A Hosp. } \\
(n=6)\end{array}$ & $\begin{array}{l}\text { B Hops. } \\
(n=7)\end{array}$ & $\begin{array}{l}\text { C Hosp. }(n= \\
7)\end{array}$ & $\begin{array}{l}\text { D Hosp. } \\
(n=9)\end{array}$ \\
\hline \multicolumn{6}{|l|}{ Demographics } \\
\hline Age, years, mean (SD) & $75(9)$ & $78(6)$ & $74(6)$ & $70(8)$ & $80(11)$ \\
\hline Sex (male), n (\%) & $20(69)$ & $4(67)$ & $5(71)$ & $5(71)$ & $6(67)$ \\
\hline Atrial fibrillation, n (\%) & $21(72)$ & $5(83)$ & $5(71)$ & $6(86)$ & $5(56)$ \\
\hline Hypertension , n (\%) & $19(66)$ & $5(83)$ & $5(71)$ & $4(57)$ & $5(56)$ \\
\hline Dyslipidemia , n (\%) & $11(38)$ & $1(17)$ & $3(43)$ & $5(71)$ & $2(22)$ \\
\hline Diabetes mellitus , $\mathrm{n}(\%)$ & $7(24)$ & 0 & $3(43)$ & $2(29)$ & $1(11)$ \\
\hline \multicolumn{6}{|l|}{ Clinical and imaging features } \\
\hline IV-tPA, n (\%) & $17(59)$ & $4(67)$ & $5(71)$ & $3(43)$ & $5(56)$ \\
\hline Baseline mRS, median (IQR) & $0(0-1)$ & $1(0-1)$ & $1(0-2)$ & $0(0-1)$ & $0(0-0)$ \\
\hline Poor baseline mRS, $\geq 3, \mathrm{n}(\%)$ & $5(17)$ & 0 & $2(29)$ & $1(14)$ & $2(22)$ \\
\hline NIHSS, median (IQR) & $19(16-24)$ & $22(19-26)$ & $14(11-20)$ & $19(18-22)$ & $22(18-26)$ \\
\hline DWI-ASPECTS, median (IQR) & $7(5-9)$ & $8(7-9)$ & $7(6-9)$ & $8(5-10)$ & $5(4-6)$ \\
\hline \multicolumn{6}{|l|}{ Occlusion site, n (\%) } \\
\hline ICA & $12(41)$ & $2(33)$ & $4(57)$ & $2(29)$ & $3(33)$ \\
\hline M1 & $11(38)$ & $3(50)$ & $3(43)$ & $3(43)$ & $3(33)$ \\
\hline M2 & $3(10)$ & $1(17)$ & 0 & 0 & $2(22)$ \\
\hline VA/BA & $3(10)$ & 0 & 0 & $2(29)$ & $1(11)$ \\
\hline
\end{tabular}

DWI-ASPECTS: diffusion-weighed image-Alberta Stroke Programme Early CT Score; IV-tPA: intravenous tissue plasminogen activator; mRS: modified Rankin Scale; NIHSS: National Institute of Health Stroke Scale

results and significance, considering that it should be switched to the "Drip and Ship (or Mothership)" system considering the individual circumstances.

We expected that the treatment results at Hospital A, which is located in another prefecture, and requires approximately 60 minutes of travel, would to be worse than those at Hospital D, which is located within the city and is a mere 20 minutes by car. However, actual results were contrary to our expectation. Concerning the interval until recanalization, in Hospital D, there was a shorter mean onset-torecanalization time (ORT) (229 minutes) than the other institutions given that devices were already equipped and endovascular treatment specialists arrived promptly, as reflected by the geographic circumstances. On the other hand, Hospital A had the longest distance from our hospital and insufficiently equipped devices, resulting in a mean ORT of 393 minutes. Although both hospitals had similar median NIHSS scores (i.e., 22), Hospital D also treated patients with a low DWI-ASPECTS, leading to a lower proportion of patients with a favorable mRS score than that at Hospital A. At Hospital D, treatment was increasingly indicated even for patients with a low DWI-ASPECTS on the assumption that some may be reversed through prompt recanalization due to the short distance, which may explain the marked difference from Hospital A.

As presented in Table 3, univariate analysis also demonstrated that the DWI-ASPECTS the most markedly influenced the prognosis. In the guidelines for the adequate use of devices for percutaneous transluminal mechanical thrombectomy (3rd version, March 2018) in Japan, this procedure is strongly recommended for patients with a DWI-ASPECTS of $\geq 7$ as Grade A. This is consistent with the results of our series. Therefore, currently, when adopting the "Drip and Go" system in our department, the indication of treatment is carefully reviewed for patients with a DWI-ASPECTS of $\leq 6$, emphasizing the DWI-ASPECTS rather than the distance from our hospital. Until cooperation/manpower for acute-phase AIS treatment per secondary medical area is sufficiently arranged, we will continue the "Drip and Go" inter-hospital cooperation system through adequate patient selection.

Several studies that compared trip treatment by a mobile stroke team, termed "Trip and Treat" or "Drip and Drive" systems, with direct transportation, ${ }^{19-21)}$ which are similar 
Table 2 Summary of the procedural features, workflow time, and neurological outcome at each hospital

\begin{tabular}{|c|c|c|c|c|c|}
\hline & $\begin{array}{l}\text { All cases } \\
(\mathrm{n}=29)\end{array}$ & $\begin{array}{l}\text { A Hosp. } \\
(n=6)\end{array}$ & $\begin{array}{l}\text { B Hops. } \\
(n=7)\end{array}$ & $\begin{array}{l}\text { C Hosp. } \\
(n=7)\end{array}$ & $\begin{array}{l}\text { D Hosp. } \\
(n=9)\end{array}$ \\
\hline Device stand-by & & None & None & Yes & Yes \\
\hline \multicolumn{6}{|l|}{ Procedural features } \\
\hline Combined (SR + Aspiration), n (\%) & $23(79)$ & $6(100)$ & $7(100)$ & $2(28)$ & $8(89)$ \\
\hline O2P, min, mean (SD) & $259(90)$ & $288(40)$ & $296(88)$ & $235(102)$ & $221(87)$ \\
\hline D2P, min, mean (SD) & $186(79)$ & $222(52)$ & $210(80)$ & $156(93)$ & $170(65)$ \\
\hline P2R, min, mean (SD) & $68(35)$ & $104(50)$ & $59(17)$ & $61(25)$ & $58(20)$ \\
\hline ORT, min, mean (SD) & $313(119)$ & $393(73)$ & $354(128)$ & $312(101)$ & 229 (123) \\
\hline Final mTICI 2b+3, n (\%) & $24(83)$ & $5(83)$ & $6(86)$ & $5(71)$ & $8(89)$ \\
\hline Number of passes, median (IQR) & $2(1-2)$ & $2(1-2)$ & $1(1-2)$ & $2(2-3)$ & $1(1-2)$ \\
\hline First-pass recanalization, n (\%) & $14(48)$ & $3(50)$ & $4(57)$ & $2(29)$ & $5(56)$ \\
\hline Hemorrhagic complication, n (\%) & $1(3)$ & $1(17)$ & 0 & 0 & 0 \\
\hline \multicolumn{6}{|l|}{ Outcomes (at 90 day, or discharge) } \\
\hline$m R S \geq 2, n(\%)$ & $6(21)$ & $3(50)$ & $1(14)$ & $1(14)$ & $1(11)$ \\
\hline $\mathrm{mRS} \geq 3, \mathrm{n}(\%)$ & $12(41)$ & $4(67)$ & $3(43)$ & $3(43)$ & $2(22)$ \\
\hline mRS deterioration, 2 or less, $\mathrm{n}(\%)$ & $11(38)$ & $4(67)$ & $3(43)$ & $2(29)$ & $2(22)$ \\
\hline
\end{tabular}

D2P: door-to-puncture time; mRS: modified Rankin Scale; O2P: onset-to-puncture time; ORT: onset-to-reperfusion time; P2R: puncture-to-reperfusion time; SR: stent retreiver

to our own reported that physician trip thrombectomy group had significantly shorter imaging to puncture time than patient transfer group within $50-$ to $60-\mathrm{km}$ areas and urban areas. The furthest hospital included in the current study was also located approximately $60-\mathrm{km}$ away, requiring approximately 1 hour for transfer by car. Osanai et al. established an inter-hospital cooperation system involving 1-hour drive areas in Hokkaido (drive and retrieve method), wherein trip thrombectomy by neuroendovascular treatment specialists was performed, reported median ORT of 229 minutes and a median D2P of 80 minutes. They reported that many patients with AIS were covered in a short time, leading to favorable results. ${ }^{22}$ ) This may have resulted from efforts to shorten the interval until thrombectomy as much as possible at respective medical institutions participating in the cooperative system in Hokkaido. According to previous studies, a short interval until puncture significantly increases the rate of patients with favorable recanalization ${ }^{23)}$ and the functional prognosis reduces with a delay in the timing of puncture ${ }^{13,24)}$; the interval until puncture is an important prognostic factor.

One limitation of the current series was that the median D2P was approximately $180 \mathrm{~min}$, causing a time loss. In the future, educational activities for each medical institution and a critical workflow must be arranged to shorten the time taken until mechanical thrombectomy is performed through prompt/close cooperation. Indeed, in 2019, minimum devices for mechanical thrombectomy were equipped in Hospital A and devices/aspiration pumps were installed in primary cooperative institutions for the "Drip and Go" system. In addition, we recommended that preparations for puncture be made before the specialists arrive. Currently, our department has established no cooperation with remote hospitals requiring a transfer time of $\geq 60$ minutes from our hospital. In such cases, the "Drip and Ship/Mothership" systems are recommended, as previously conducted.

\section{Limitations of the "Drip and Go" system and this study}

One limitations of the present study are that among the patients referred to our department for consultation, physicians in charge of stroke at the affiliated hospitals considered the possibility of mechanical thrombectomy indication; there was a selection bias. In addition, this was a retrospective observation study with low data reliability and a limited number of patients for statistical analysis. In the future, patients in 2019 will be additionally analyzed to extract issues. Furthermore, a support system to provide prompt, high-quality treatment should be established by sharing information with the physicians and staff belonging to the affiliated hospitals, and holding study meetings. 


\begin{tabular}{|c|c|c|c|}
\hline & $\begin{array}{l}\mathrm{mRS} 0-3 \\
(\mathrm{n}=12)\end{array}$ & $\begin{array}{c}\mathrm{mRS} 4-6 \\
(\mathrm{n}=17)\end{array}$ & $\mathrm{P}$ value ${ }^{\dagger}$ \\
\hline \multicolumn{4}{|l|}{ Variables } \\
\hline Age (under 80 years old), n (\%) & $10(83)$ & $10(59)$ & 0.32 \\
\hline Baseline mRS <3, n (\%) & $11(92)$ & $13(76)$ & 0.57 \\
\hline NIHSS <26, n (\%) & $11(92)$ & $9(53)$ & 0.07 \\
\hline O2P <270 min, n (\%) & $5(42)$ & $10(59)$ & 0.59 \\
\hline $\mathrm{O} 2 \mathrm{P}<438 \min , \mathrm{n}(\%)$ & $11(92)$ & $15(88)$ & 1.00 \\
\hline P2R <60 min, $\mathrm{n}(\%)$ & $5(42)$ & $10(59)$ & 0.59 \\
\hline DWI-ASPECTS $\geq 8, \mathrm{n}(\%)$ & $7(58)$ & $5(29)$ & 0.24 \\
\hline DWI-ASPECTS $\geq 7, \mathrm{n}(\%)$ & $12(100)$ & $7(41)$ & $<0.01$ \\
\hline DWI-ASPECTS $\geq 6, \mathrm{n}(\%)$ & $12(100)$ & $9(53)$ & 0.02 \\
\hline
\end{tabular}

\section{Conclusion}

Although the usefulness of mechanical thrombectomy for AIS had been previously established, regional differences in medical sources and patients who do not benefit therefrom are still present. Even in areas/institutions where patient transfer to TSCs or CSCs may be difficult to arrange in the future, mechanical thrombectomy using the "Drip and Go" system may provide stable treatment outcome in adequately selected patients within a 1-hour drive areas. This may be a strategy to cover areas without specialists in neuroendovascular treatment for the further widespread application of mechanical thrombectomy.

\section{Acknowledgments}

We thank Drs. Koketsu (Department of Neurosurgery, Tosei General Hospital), Mizutani (Department of Neurosurgery, Konan Kosei Hospital), Tsujiuchi (Department of Neurosurgery, Daido Hospital), and Inoue (Department of Neurosurgery, Chuno Kosei Hospital) for their cooperation.

\section{Disclosure Statement}

Takashi Izumi received a research grant from KANEKA MEDIX CORP. The other authors declare no conflicts of interest.

\section{References}

1) The Japan Stroke Society, The Japan Neurosurgical Society, The Japanese Society for Neuroendovascular Therapy. Guidelines for the Proper Use of Percutaneous Transluminal Cerebral Thrombus Retrieval Devices, Edition 3. Jpn J Stroke 2018; 40: 285-309. (in Japanese)

2) Yamagami H, Tanaka K, Yoshimoto $T$, et al: Current status and future aspects of mechanical thrombectomy for acute ischemic stroke. Jpn J Neurosurg 2019; 28: 552-560. (in Japanese)

3) Khatri P, Abruzzo T, Yeatts SD, et al: Good clinical outcome after ischemic stroke with successful revascularization is time-dependent. Neurology 2009; 73: 1066-1072.

4) Saver JL, Goyal M, van der Lugt A, et al: Time to treatment with endovascular thrombectomy and outcomes from ischemic stroke: a meta-analysis. JAMA 2016; 316: 1279-1288.

5) Kansagra AP, Wallace AN, Curfman DR, et al: Streamlined triage and transfer protocols improve door-to-puncture time for endovascular thrombectomy in acute ischemic stroke. Clin Neurol Neurosurg 2018; 166: 71-75.

6) Aghaebrahim A, Granja MF, Agnoletto GJ, et al: Workflow optimization for ischemic stroke in a community-based stroke center. World Neurosurg 2019; 129: e273-e278.

7) Park MS, Yoon W, Kim JT, et al: Drip, ship, and on-demand endovascular therapy for acute ischemic stroke. PLoS One 2016; 11: e0150668.

8) Hiyama N, Yoshimura S, Shirakawa M, et al: safety and effectiveness of drip, ship, and retrieve paradigm for acute ischemic stroke: a single center experience. Neurol Med Chir (Tokyo) 2016; 56: 731-736. 
9) Weber R, Reimann G, Weimar C, et al: Outcome and periprocedural time management in referred versus directly admitted stroke patients treated with thrombectomy. Ther Adv Neurol Disord 2016; 9: 79-84.

10) Ismail M, Armoiry X, Tau N, et al: Mothership versus drip and ship for thrombectomy in patients who had an acute stroke: a systematic review and meta-analysis. J Neurointerv Surg 2019; 11: 14-19.

11) McTaggart RA, Ansari SA, Goyal M, et al: Standards and Guidelines Committee of the Society of NeuroInterventional Surgery (SNIS): initial hospital management of patients with emergent large vessel occlusion (ELVO): report of the standards and guidelines committee of the Society of NeuroInterventional Surgery. J Neurointerv Surg 2017; 9: 316-323.

12) Goyal M, Menon BK, van Zwam WH, et al: Endovascular thrombectomy after large-vessel ischaemic stroke: a meta-analysis of individual patient data from five randomised trials. Lancet 2016; 387: 1723-1731.

13) Jahan R, Saver JL, Schwamm LH, et al: Association between time to treatment with endovascular reperfusion therapy and outcomes in patients with acute ischemic stroke treated in clinical practice. JAMA 2019; 322: 252-263.

14) Berkhemer OA, Fransen PS, Beumer D, et al: A randomized trial of intraarterial treatment for acute ischemic stroke. $N$ Engl J Med 2015; 372: 11-20.

15) Schlemm E, Ebinger M, Nolte $\mathrm{CH}$, et al: Optimal transport destination for ischemic stroke patients with unknown vessel status: use of prehospital triage scores. Stroke 2017; 48: 2184-2191.

16) Froehler MT, Saver JL, Zaidat OO, et al: Interhospital transfer before thrombectomy is associated with delayed treatment and worse outcome in the STRATIS Registry (systematic evaluation of patients treated with neurothrombectomy devices for acute ischemic stroke). Circulation 2017; 136: 2311-2321.

17) Mendez B, Requena $M$, Aires A, et al: Direct transfer to angio-suite to reduce workflow times and increase favorable clinical outcome. Stroke 2018; 49: 2723-2727.

18) Zaidat OO, Haussen DC, Hassan AE, et al: Impact of stent retriever size on clinical and angiographic outcomes in the STRATIS Stroke Thrombectomy Registry. Stroke 2019; 50: 441-447.

19) Amano $T$, Sato $M$, Teranishi $Y$, et al: Comparison of a novel inter-hospital system "Mobile Endovascular Therapy Team" and patient transfer system in mechanical thrombectomy for acute ischemic stroke. JNET J Neuroendovasc Ther 2015; 9: 238-244.

20) Brekenfeld C, Goebell E, Schmidt H, et al: 'Drip-anddrive': shipping the neurointerventionalist to provide mechanical thrombectomy in primary stroke centers. $J$ Neurointerv Surg 2018; 10: 932-936.

21) Wei D, Oxley TJ, Nistal DA, et al: Mobile interventional stroke teams lead to faster treatment times for thrombectomy in large vessel occlusion. Stroke 2017; 48: 3295-3300.

22) Osanai $T$, Ito $Y$, Ushikoshi $S$, et al: Efficacy of 'drive and retrieve' as a cooperative method for prompt endovascular treatment for acute ischemic stroke. J Neurointerv Surg 2019; 11: 757-761.

23) Bourcier R, Goyal M, Liebeskind DS, et al: Association of time from stroke onset to groin puncture with quality of reperfusion after mechanical thrombectomy: a meta-analysis of individual patient data from 7 randomized clinical trials. JAMA Neurol 2019; 76: 405-411.

24) Mueller-Kronast NH, Zaidat OO, Froehler MT, et al: Systematic evaluation of patients treated with neurothrombectomy devices for acute ischemic stroke: primary results of the STRATIS Registry. Stroke 2017; 48: 2760-2768. 